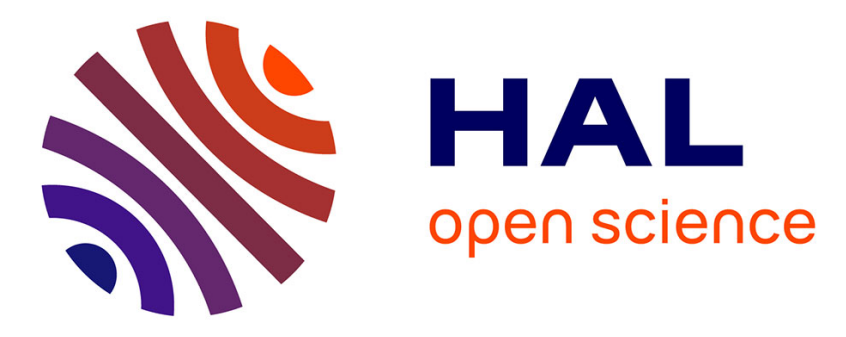

\title{
CT-based semi-automatic quantification of vertebral fracture restoration
}

\author{
Gilles Le Pennec, Sophie Campana, Erwan Jolivet, Jean-Marc Vital, Xavier \\ Barreau, Wafa Skalli
}

\section{- To cite this version:}

Gilles Le Pennec, Sophie Campana, Erwan Jolivet, Jean-Marc Vital, Xavier Barreau, et al.. CT-based semi-automatic quantification of vertebral fracture restoration. Computer Methods in Biomechanics and Biomedical Engineering, 2012, 17 (10), pp.1086-1095. 10.1080/10255842.2012.736968 . hal03264706

\section{HAL Id: hal-03264706 https://hal.science/hal-03264706}

Submitted on 18 Jun 2021

HAL is a multi-disciplinary open access archive for the deposit and dissemination of scientific research documents, whether they are published or not. The documents may come from teaching and research institutions in France or abroad, or from public or private research centers.
L'archive ouverte pluridisciplinaire HAL, est destinée au dépôt et à la diffusion de documents scientifiques de niveau recherche, publiés ou non, émanant des établissements d'enseignement et de recherche français ou étrangers, des laboratoires publics ou privés. 


\title{
CT-based semi-automatic quantification of vertebral fracture restoration
}

\author{
Gilles Le Pennec $^{\mathrm{a} 1} *$, Sophie Campana ${ }^{\mathrm{a} 2}$, Erwan Jolivet ${ }^{\mathrm{a} 3}$, Jean-Marc Vital ${ }^{\mathrm{b}}$, Xavier Barreau ${ }^{\mathrm{c} 5}$ and Wafa Skallia ${ }^{\mathrm{a}}$ \\ ${ }^{a}$ Arts et Metiers ParisTech, LBM, 151 bd de l’hopital, Paris 75013, France; ${ }^{b}$ Adults Emergency, CHU Bordeaux, Place Amélie Raba- \\ Léon, Bordeaux 33000, France; ${ }^{c}$ Neuroradiology, CHU Bordeaux, Place Amélie Raba-Léon, Bordeaux 33000, France
}

\begin{abstract}
Minimally invasive surgeries aiming to restore fractured vertebral body are increasing; therefore, our goals were to create a 3D vertebra reconstruction process and design clinical indices to assess the vertebral restoration in terms of heights, angles and volumes. Based on computed tomography (CT)-scan of the vertebral spine, a 3D reconstruction method as well as relevant clinical indices were developed. First, a vertebra initial solution requiring 5 min of manual adjustments is built. Then an image processing algorithm places this solution in the CT-scan images volume to adjust the model's nodes. On the vertebral body's anterior and posterior parts, nine robust heights, volume and endplate angle measurement methods were developed. These parameters were evaluated by reproducibility and accuracy studies. The vertebral body reconstruction accuracy was $1.0 \mathrm{~mm}$; heights and volume accuracy were, respectively, 1.2 and $179 \mathrm{~mm}^{3}$. In conclusion, a 3D vertebra reconstruction process requiring little user time was proposed as well as 3D clinical indices assessing fractured and restored vertebra.
\end{abstract}

Keywords: vertebrae; assessment; 3D reconstruction; semi-automatic; clinical indices; surgical restoration

\section{Introduction}

Large occurrence of vertebral fractures remains a critical medical concern, with 700,000 annual cases, due to osteoporosis in the USA (Riggs and Melton 1995). In Europe, 1.4 million persons in the 50-79 years of age range may annually develop new spinal injuries (Felsenberg et al. 2002), with devastating clinical impact on the quality of life (Gold 1996). Several surgical methods exist to restore vertebral height, such as vertebroplasty and kyphoplasty. The objective and quantitative evaluation of the resulting vertebral restoration is essential in order to provide a reliable patient-specific estimation of the morphometric changes induced by surgery. However, such assessments raise major issues; indeed, the National Osteoporosis Foundation Working Group underlined the difficulty to measure reliable vertebral body dimensions on 2D sagittal radiographs. Genant et al. (1993) proposed a 2D X-ray methodology based on the placement of six points per vertebra, defining anterior, middle and posterior sagittal heights; unfortunately, these measurements cannot provide information about the right or left heights of the vertebral body; despite providing acceptable agreement between operators, these measurements still show a significant variability in the inter-observer results (Genant et al. 1993). This reproducibility issue is among others related to the intrinsic 2D radiography characteristics, i.e. the conical $\mathrm{X}$-ray projection combined to the superimposition of tissues and bony structures. The image qualities resulting from the sensitivity to the radiographic equipment in the case of multi-centric studies are also a factor for obtaining unsatisfactory reproducible results. However, Genant's semi-quantitative method remains recommended and currently used in clinical routine, as no more effective alternative exists (Grados et al. 2009). Under these conditions, the handling of a $3 \mathrm{D}$ vertebra could be interesting to overcome bias generated by $2 \mathrm{D}$ examination: the use of computed tomography (CT)-scan would provide a much more accurate assessment of vertebral dimensional criteria such as volumes or angles or region of interest between the endplates. Nevertheless, 3D reconstruction starting from CT-scan slices is a tedious and time-consuming work, as developing reliable semi-automatic segmentation software remains an issue. Several tools dedicated to this application have been recently proposed (Kaminsky et al. 2004; Mastmeyer et al. 2006; Klinder et al. 2009), but they still require long processing times with an insufficient accuracy. Moreover, these attempts have never been applied to the reconstruction of a fractured vertebra. More recently, Laurent et al. (2011) developed a semi-automated method for the 3D reconstruction of human cranial vault, based on the parametric description of the shape to be reconstructed, and on the identification of anatomical landmarks used in the initialisation stage. For the purpose of this study, this software was adapted to the $3 \mathrm{D}$ reconstruction of fractured vertebrae, allowing the exploitation of a 3D geometry for investigating relevant metrical parameters. Therefore, the aim of this work was to propose and validate a methodology for quantitative estimation of vertebral restoration,

*Corresponding author. Email: gilles.lepennec@ensam.eu 
based on 3D reconstruction from CT-scan obtained with a semi-automated segmentation process.

\section{Materials and methods}

\section{$2.13 D$ reconstruction}

\subsubsection{Semi-automatic reconstruction}

The semi-automatic treatment developed to obtain the 3D geometry of a vertebra was based on sequential algorithmic steps as described below:

(1) The CT-scan slices were processed using a ray casting method (Roth 1982) in order to generate Digital Reconstructive Radiograph (DRR) images (Figure 1).

(2) Using the DRR images, an initial 3D solution made of a 3D geometric surface of the vertebra was created according to the method proposed by Humbert et al. (2009).

(3) Starting from the 3D model of the initial solution, normal vectors $\vec{n}$ were defined for each vertex according to the polygons' normal in its neighbourhood. Along $\vec{n}$ normals, intensity profiles were computed in the CT-scan images volume to detect at best the cortical position; the node position was then adjusted. As a result, the initial solution 3D shape was adjusted along its nodes' normal through multiple research of the cortical position in the CTscan images volume. This computing, derived from

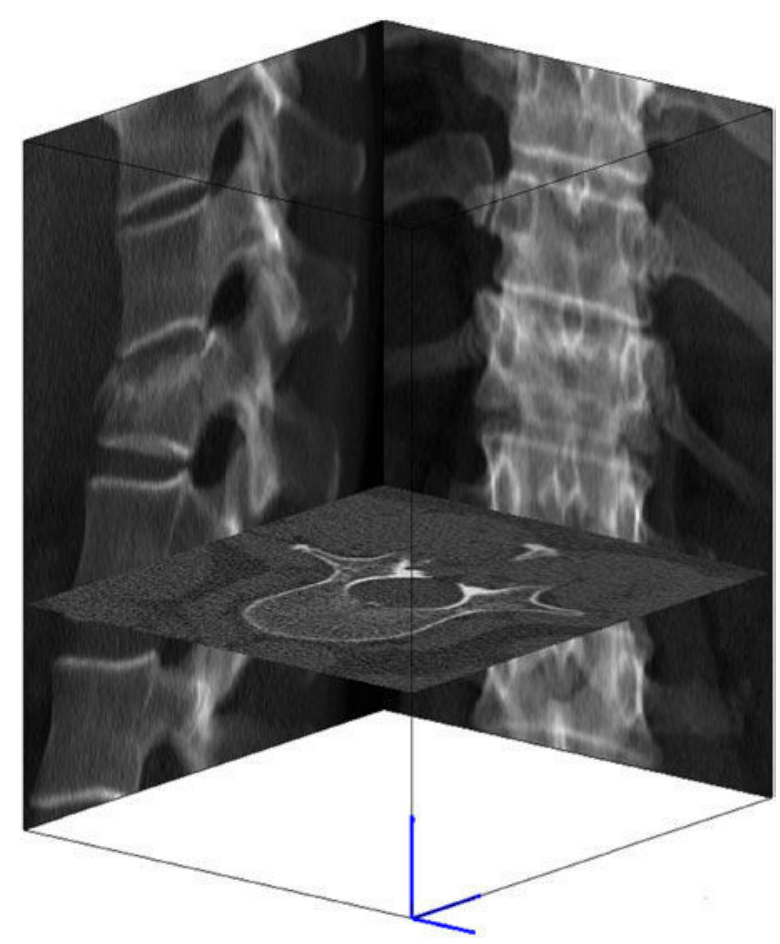

Figure 1. Frontal and sagittal DRR generation from CT-scan. the study of Laurent et al. (2011), was done using the addition of coherence criteria to avoid aberrant pits on the surface.

\subsubsection{Frame associated with the $3 D$ reconstruction}

For each vertebra, a frame of reference (O, X, Y and Z) was defined using the sagittal plane, the vertebral body and the pedicles (Figure 2). The origin $\mathrm{O}$ is located in the middle of the segment defined by the barycentre of the superior endplate and the barycentre of the inferior endplate. The postero-anterior axis defined the $\mathrm{X}$ vector. The axis joining the barycentre of the inferior endplate and the superior endplate represented the $\mathrm{Z}$ vector. The last $\mathrm{Y}$ vector was located in the transverse plane and orthogonal to $\mathrm{X}$ and $\mathrm{Z}$. The mid-sagittal plane was defined as $\mathrm{O}, \mathrm{X}, \mathrm{Z}$ and the mid-frontal plane was defined as O,Y,Z.

\subsection{Quantification of clinical parameters}

\subsubsection{Generic vertebra}

In order to quantify the clinical parameters, a generic 3D model describing the specific anatomic features of the considered thoracic or lumbar level was developed. These models were generated from the average vertebrae of a database of 121 spines constituted with thoracic vertebrae by Laporte et al. (2000) and with lumbar vertebrae by Semaan et al. (2001).

\subsubsection{Pre-post analysis for restoration quantification}

The restoration vertebra evolution is defined by comparison between the fractured and the surgical restored status. The comparison between two 3D solutions was based on the hypothesis that after a minimally invasive surgery

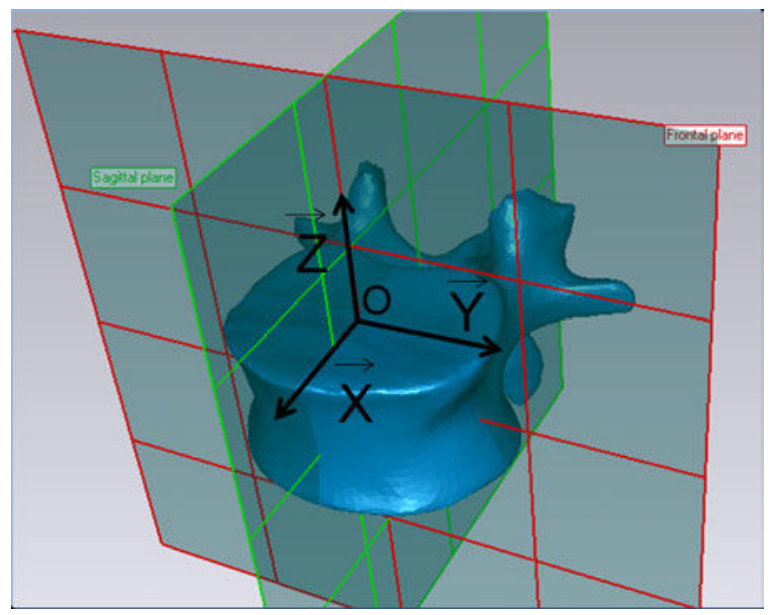

Figure 2. Anatomical frame $(\mathrm{O}, \mathrm{X}, \mathrm{Y}, \mathrm{Z})$ associated with the vertebrae. 


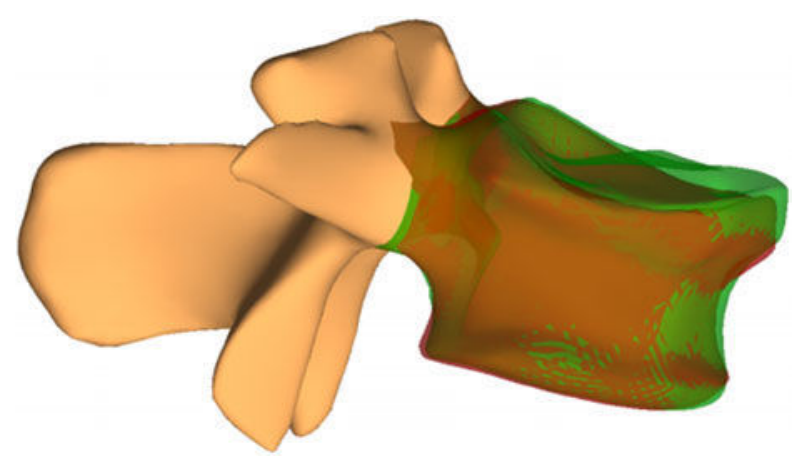

Figure 3. Post-surgical placed over pre-surgical vertebra for restoration estimation.

located on the vertebral body, the posterior part of the vertebra remains unchanged. Using this criterion, a best-fit rigid registration of the restored vertebra was made on the fractured vertebta using the posterior arch (Figure 3). Then the differences were calculated for each parameter.

\subsubsection{Height measurement}

In order to evaluate the height restoration of each vertebra after surgery, two grids each composed of nine points were used and precisely defined on the surfaces of the superior and inferior endplates, respectively, allowing nine local height calculations. The locations of these 18 points were first defined on each generic vertebra, and then morphed on the specific vertebra to be measured. Steps are given in the following.

(1) Placement of the nine points on the superior endplate.

First, by moving the mid-frontal plane along the mid-sagittal plane, two extremity points were determined on the anterior and posterior parts of the vertebral body (Figure 4); two anterior and posterior frontal planes were thus obtained, which were shifted by $3 \mathrm{~mm}$ towards the inner vertebral body to avoid the irregularities. The intersection between the two shifted frontal planes and the mid-sagittal plane provides two anterior (Ref1) and posterior (Ref2) points, projected on the superior endplate. The middle of the segment joining these two points creates a 3rd point (Ref3). Next, to compute the left and right points of the grid, the mid-sagittal plane was shifted to tangent the inner border of the pedicles: two right and left sagittal planes were obtained; their intersection with the posterior frontal plane results in two posterior right (Ref4) and left (Ref5) points, which are thus aligned. The mid-frontal plane was then shifted towards the anterior direction, along the left sagittal plane, until intersection with the border of the vertebral body was found; a $3 \mathrm{~mm}$ shift was applied to this point in the posterior direction, giving the anterior left point (Ref6) projected onto the endplate.

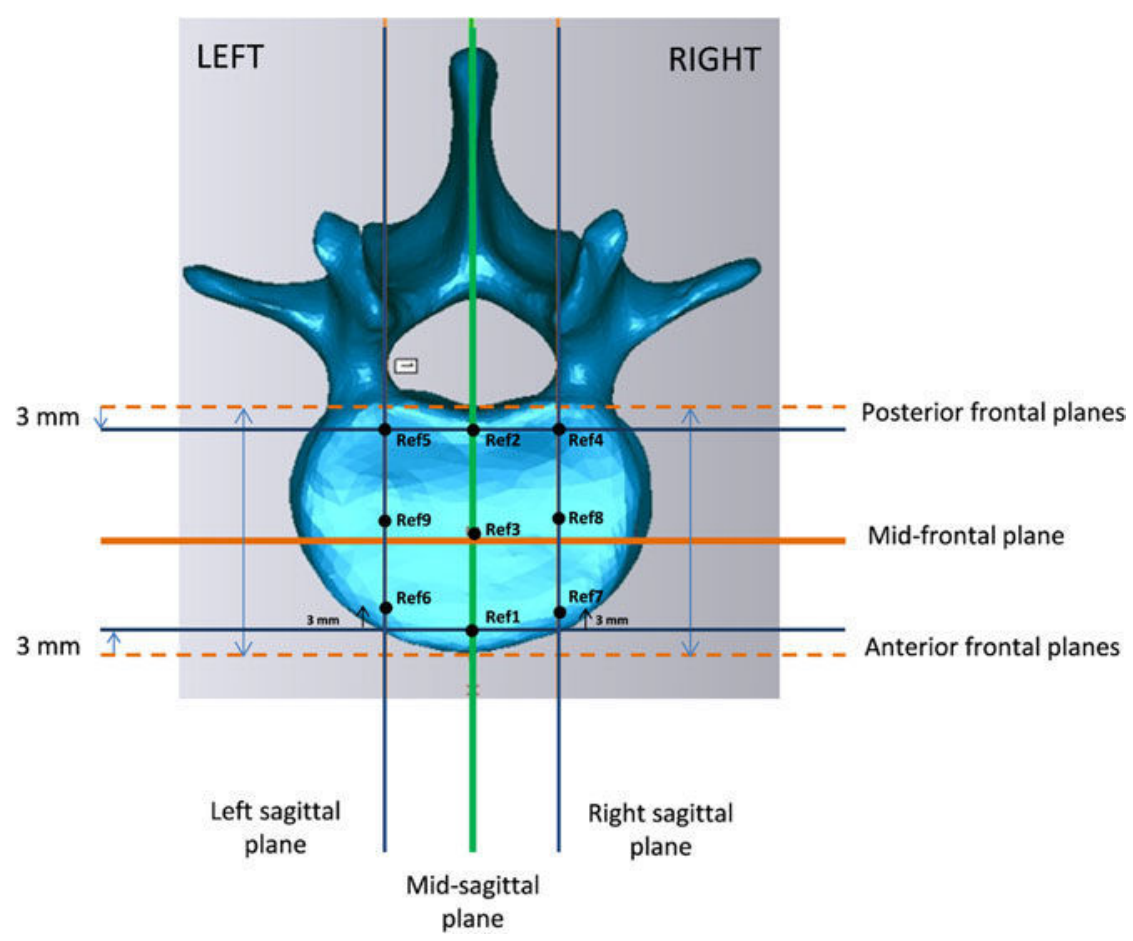

Figure 4. Grid construction: Right and left sagittal planes tangent the inner border of the pedicles; Anterior and posterior frontal planes, before the 3-mm shift, tangent the extremities of the vertebral body. 
The same process was followed on the right anterior side of the vertebra to compute the anterior right point (Ref7). Finally, middle of Ref7-Ref4 and middle of Ref5-Ref6 vertically projected on the surface provided the points Ref8 and Ref9.

(2) Placement of the nine points on the inferior endplate.

Likewise, the same process was followed as for the superior grid to obtain nine points located on the inferior endplate of the vertebra.

(3) Deformation function for the grid.

Once the 18 points on the inferior and superior grids were obtained on the generic vertebra, a deformation function was computed to adapt these grids to the reconstructed vertebra. This deformation function was a kriging function using 5000 control points of the generic vertebra and the corresponding points on the semi-automatic reconstructed vertebra. The height computation can hence be done on the semi-automatic grids.

(4) Height computation.

To calculate the nine local heights of the vertebra at the nine locations of the grid, two options were considered.

(1) Nine Euclidean distances were calculated between the nine points of the superior grid and the nine corresponding points of the inferior grid. Inferior and superior least square planes based on both grids are computed: the points of each grid are then projected on the corresponding plane (Figure 5).

(2) The heights were finally obtained using the Euclidean distances between the associated inferior and superior projected points.

\subsubsection{Volume measurement}

To quantify the volume, a vertebral core represented by an extruded ellipse was calculated (Figure 6). Two options were considered to construct this ellipse with the 3D model of the reconstructed vertebra. Both these options compute a vertebral body volume: the first option focuses on the vertebral core without the irregularities of the

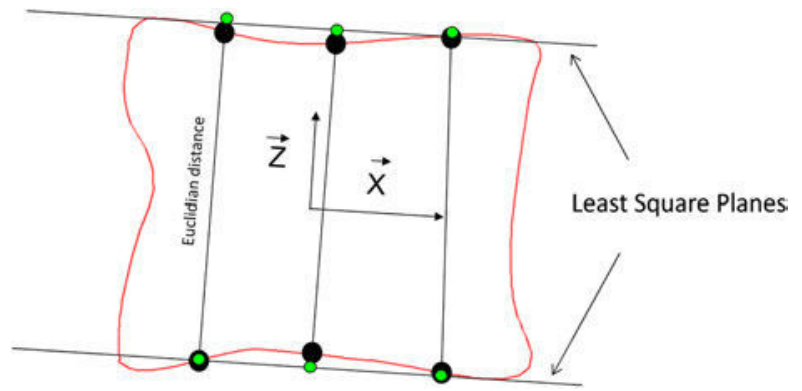

Figure 5. Height measurement (sagittal view) using points located on the endplates or projected on least square planes.

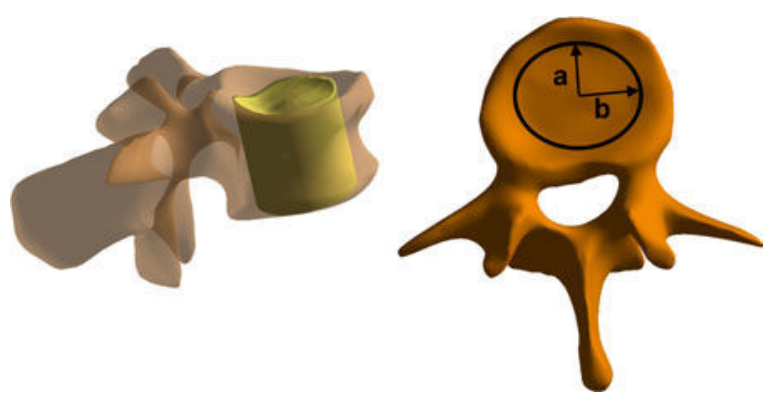

Figure 6. Extruded ellipse for the calculation of volume.

borders while the second option represents the overall body volume as described below.

Ellipse 1: On each endplate, the polygons describing the external borders were researched. It was performed by selecting the polygons located at the intersection of the vertebral wall and the endplate. Using the frame of reference, the borders of the superior and inferior endplate were projected onto the transverse plane. A least square ellipse (Fitzgibbon et al. 1999) was then estimated on the results of this projection. Because the borders of the superior vertebral endplate are not continuous, the ellipse is reduced in its two diameters by $20 \%$.

Ellipse 2: The second ellipse was constructed with eight points. On the superior and inferior grid, the middle anterior points and middle posterior points were selected. To obtain the left and right points on each plate, a frontal plane was placed at the middle-middle point of the grids. The intersection between this plane and the vertebra's surface provides a surface section, where the extreme points were selected at each side. The four resulting points were then shifted by $3 \mathrm{~mm}$ in the section's axis. Using the frame of reference, the superior and inferior points selected were projected onto the transverse plane where the extrusion ellipse was evaluated using the least square method. Once the ellipse was computed, an extrusion was made in the inferior and superior direction according to the vertical direction of the frame of reference. The extrusion process computed the projection on the $3 \mathrm{D}$ surface of cylinder.

\subsubsection{Local heights and volumes}

In order to measure the local restoration on the vertebral endplates, the grids yielded definition of nine regions of interest on each endplate (Figure 7). To build these rectangular areas on the inferior and superior endplates, the anterior border is defined using the grids' anterior left and right points. The lateral side length was defined by the position of the middle posterior points. This area was divided into three by three equal rectangular zones, which results in nine rectangular areas on the inferior and superior endplates. These regions were projected on the 


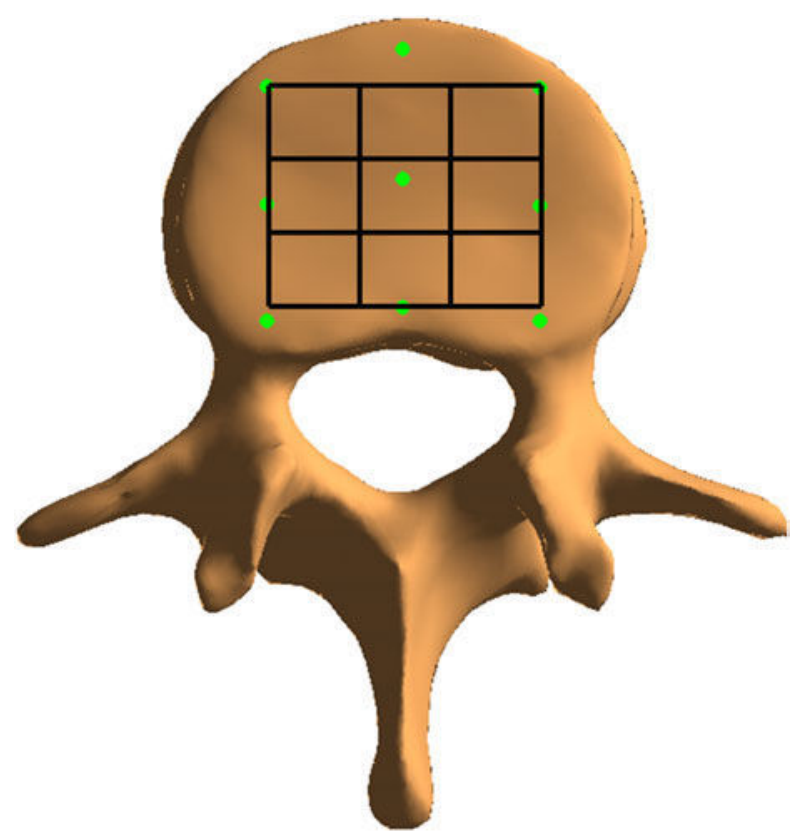

Figure 7. Location of the nine zones according to the grid.

vertebra surface. Finally, the corner nodes of the corresponding inferior and superior zones were connected to form nine volumes. For each volume the average height, the maximal height and the volume can be computed. These nine zones can also be called regions of interest of the vertebral body.

\subsubsection{Angle measurement}

The angle measurement was made on the vertebrae by computing least square planes on each grid. The 3D angle, sagittal and frontal angles between both normal of the inferior and superior planes were then calculated (Figure 8). The sagittal angle is the angle formed in the sagittal plane between the least square planes. Likewise, frontal angle is formed in the frontal plane between the least square planes.

\subsection{Method evaluation}

\subsubsection{Manual reference evaluation}

Manual reconstructed vertebrae were used as a reference for the evaluation of this semi-auto method. This manual reference itself was evaluated in a preliminary step. Reconstructions were manually processed using AVIZO software, able to generate after segmentation a 3D model of the vertebra composed of 30,000 polygons. The reproducibility study was evaluated: three different welltrained operators reconstructed two pre- and post-surgical vertebrae (slice thicknesses $=1 \mathrm{~mm}$; gap between slices $=0.5 \mathrm{~mm}$ ); one operator reconstructed the vertebrae twice. Point-to-surface differences were evaluated between all these reconstructions. As a result, both interand intra-observer $95 \%$ confidence intervals were under $0.5 \mathrm{~mm}$. The resulting vertebrae could thus be considered as gold standard (GS) data, and used as a comparison basis for the $3 \mathrm{D}$ vertebrae generated with the semi-automatic software.

\subsubsection{Clinical parameters accuracy study}

In the accuracy study, 12 lumbar and 4 thoracic vertebrae from 16 patients with spinal surgery (SpineJack ${ }^{\circledR}$ implant, VEXIM) were considered. The patients are composed of eight men and eight women with a mean age of 57 years with a minimal age of 21 years and a maximal age of 82 years. The 3D models were obtained from CT-scan slices (thickness between 0.675 and $1 \mathrm{~mm}$ ), which were processed in two different manners: semi-automatic and manual. The quantification of the clinical parameters was done in the first step on the semi-automatic vertebrae. In the second step, the clinical parameters were quantified on the vertebrae of reference.

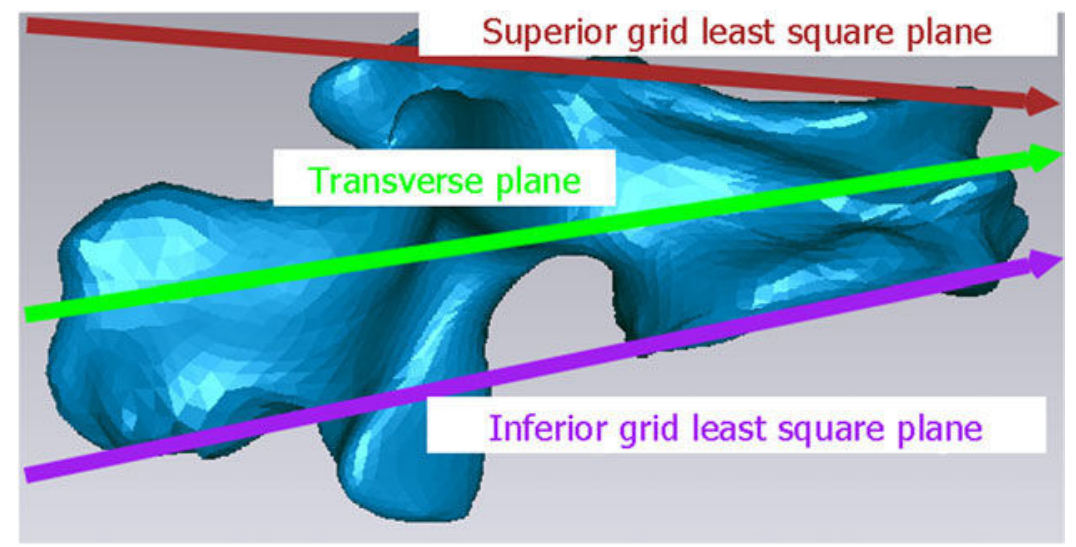

Figure 8. Grids least square planes on the vertebra. 
(1) Heights

To compute the height parameters, the semiautomatic vertebrae's grids were projected on the vertebrae of reference, by projecting the grids' points onto the surface according to the vertical direction of the frame of reference, the least square planes were then evaluated and the heights were computed.

(2) Volume.

Once the ellipse was computed and used for the extrusion on the semi-automatic vertebra, the ellipse was placed on the vertebra of reference. The ellipse was projected onto the endplate surfaces to obtain the vertebral core of reference according to the frame of reference. The volume of each of these vertebral cores was compared to evaluate the volume accuracy.

(3) Angles.

Using the least square planes computed on the vertebra of reference, the 3D, sagittal and frontal angles were computed with the same process set for the semi-automatic vertebrae.

(4) Local heights and volumes.

The nine volumes computed on the semiautomatic vertebrae were placed on the vertebrae of reference by projecting the top and bottom rectangular areas on the surface. The mean heights, maximal heights and volumes could then be evaluated on the vertebrae of reference and compared to evaluate the local heights and volumes accuracy. To evaluate these criteria, the errors of all zones were used to compute the accuracy, implying the processing of $9 \times 16$ superimpositions of pre- and post-surgery vertebrae.

\subsubsection{Statistics}

(1) Semi-automatic vertebrae shape accuracy study.

A study regarding accuracy was carried out on the 16 vertebrae data-set. The computation of 32 comparisons (16 pre-surgery and 16 post-surgery), representing a total of 114,004 surface's nodes evaluated in their point-to-surface differences, was done between semi-automatic and vertebrae of reference.

(2) Semi-automatic vertebrae clinical indices accuracy study.

For the data-set of 16 vertebrae, a systematic comparison of clinical index was made between manual and semi-automatic reconstructions. Computed values were the $95 \%$ confidence interval, the mean and the maximum of the errors.

(3) Reproducibility study of the clinical index and the shape of the semi-automatic vertebrae.

A reproducibility study was carried out on the semi-automatic reconstructions using a data-set of six vertebrae: three thoracic (T12) and three lumbar
(L1, L2, L3). For this purpose, three operators performed the reconstruction twice in both pre- and post-surgical state. The resulting data were composed of two main elements: the point-to-surface distances and the clinical parameters values. The inter- and intra-observer repeatability was computed, with the appropriate $95 \%$ confidence interval according to the Iso (1994) norm.

\subsubsection{D and $2 D$ height measurements}

To illustrate the 3D and 2D height measurement differences, these heights were compared on an L1 vertebra from our vertebrae data-set according to the study of Genant et al. (1993).

\subsection{Results}

\subsubsection{D semi-automatic reconstruction point-to-surface accuracy}

Rigid registration was made between 16 semi-automatic and 16 manually reconstructed vertebrae. The mean pointsurface distances error between reference and semiautomatic vertebral bodies was $0.26 \mathrm{~mm}$, the 2RMS (root mean square) error was $0.97 \mathrm{~mm}$ and a local maximum error value of $7.86 \mathrm{~mm}$ was located on the costal articular facet of a T12 vertebra.

\subsubsection{D semi-automatic reconstruction and clinical indices reproducibility}

Regarding reproducibility, performed on six vertebrae by three operators, results are presented in Table 1.

\subsubsection{Heights accuracy results}

Results of the heights comparison between semi-automatic and reference 3D reconstructions are presented in Table 2. Confidence interval was $1.17 \mathrm{~mm}$ without using the least square planes, and $0.92 \mathrm{~mm}$ with the least square planes. Height differences between pre- and post-surgery, representing the height restoration, were compared between semi-automatic and reference $3 \mathrm{D}$ reconstructions; these results are also presented in Table 2. The 95\% confidence interval was 1.47 and $1.19 \mathrm{~mm}$, without and with least square planes, respectively. The mean error was $-0.1 \mathrm{~mm}$ using the points located on the surface of the vertebra.

Using least square planes reduced the $95 \%$ confidence interval, which was 0.9 and $1.2 \mathrm{~mm}$, respectively, for height value and height restoration (i.e. difference prepost) value.

The difference between 3D nine grid surface heights and the nine grid heights using least square planes was 
Table 1. Reproducibility results for semi-automatic reconstruction and clinical index.

\begin{tabular}{|c|c|c|}
\hline & $\begin{array}{c}\text { Inter-observer } \\
\text { reproducibility IC 95\% }\end{array}$ & $\begin{array}{l}\text { Intra-observer } \\
\text { reproducibility }\end{array}$ \\
\hline Point-to-surface (mm) & 1.0 & 0.9 \\
\hline Grid height (mm) & 0.6 & 0.5 \\
\hline Grid height restoration $(\mathrm{mm})$ & 0.6 & 0.5 \\
\hline Ellipse volume method $1\left(\mathrm{~mm}^{3}\right)$ & 918.2 & 835.2 \\
\hline Ellipse volume restoration method 1 (mm3) & 136.7 & 128.6 \\
\hline Ellipse volume method $2\left(\mathrm{~mm}^{3}\right)$ & 1501.1 & 1454.9 \\
\hline Ellipse volume restoration method $2\left(\mathrm{~mm}^{3}\right)$ & 178.2 & 168.0 \\
\hline Heights per nine zones (mm) & 0.6 & 0.4 \\
\hline Heights restoration per nine zones (mm) & 0.7 & 0.6 \\
\hline Volume per nine zones $\left(\mathrm{mm}^{3}\right)$ & 123.0 & 93.7 \\
\hline Volume restoration per nine zones $\left(\mathrm{mm}^{3}\right)$ & 78.0 & 73.2 \\
\hline $3 \mathrm{D}$ angle restoration $\left(^{\circ}\right)$ & 2.1 & 1.9 \\
\hline Frontal angle restoration $\left({ }^{\circ}\right)$ & 1.9 & 1.7 \\
\hline Sagittal angle restoration $\left({ }^{\circ}\right)$ & 2.3 & 2.1 \\
\hline
\end{tabular}

Table 2. Height measurement accuracy.

\begin{tabular}{|c|c|c|c|c|}
\hline & IC $95 \%$ & Mean & Max & Nominal value \\
\hline Height evaluation without least square planes $(\mathrm{mm})$ & 1.2 & -0.2 & -0.2 & 23.2 \\
\hline Height evaluation using least square planes (mm) & 0.9 & 0.2 & 0.2 & 23.2 \\
\hline Height restoration evaluation without least square planes $(\mathrm{mm})$ & 1.5 & -0.1 & 2.2 & 1.4 \\
\hline Height restoration evaluation using least square planes (mm) & 1.2 & 0.1 & 1.7 & 1.4 \\
\hline
\end{tabular}

computed on the accuracy study data-set, and the mean difference was $2.1 \mathrm{~mm}(\max 8.9 \mathrm{~mm})$.

\subsubsection{Volumes accuracy results}

Volume (nominal and restored) was evaluated by using two definitions of ellipses for the generation of the vertebral core. Results are presented in Table 3 . The relative amount of volume restoration is $10 \%$ using the method 1 and $8 \%$ using the method 2.

The mean volume restoration was $1379 \mathrm{~mm}^{3}$ using the first vertebral core definition (ellipse 1) and $1513 \mathrm{~mm}^{3}$ using the second vertebral core definition (ellipse 2). The absolute mean error of the restoration evaluation was 324 and $401 \mathrm{~mm}^{3}$ for the first and the second methods, respectively.

\subsubsection{Angles accuracy results}

The angles restoration accuracy is described in Table 4.

The vertebral angle restoration accuracy was calculated, no significant differences were found between preand post-accuracy, and therefore, these values were pooled.

\subsubsection{Local evaluation accuracy results}

The precision of the nine zones used to evaluate the local restoration is presented in Table 5. These regions of interest represent the local restoration on the vertebral endplates in terms of heights and volumes. The $95 \%$ confidence interval of the average height restoration was $1.6 \mathrm{~mm}$, and the maximal restoration evaluation error was $1.7 \mathrm{~mm}$. The absolute mean error of volume restoration

Table 3. Volume measurement accuracy.

\begin{tabular}{|c|c|c|c|c|}
\hline Errors $\left(\mathrm{mm}^{3}\right)$ & Pre and Post & Restoration & $\begin{array}{l}\text { Restoration error/volume } \\
\text { restoration }(\%)\end{array}$ & $\begin{array}{c}\text { Restoration error/global } \\
\text { volume }(\%)\end{array}$ \\
\hline \multicolumn{5}{|l|}{ Method ellipse 1} \\
\hline Absolute mean & 205 & 324 & 23 & 2.4 \\
\hline $\operatorname{Max}$ & 585 & 789 & 57 & 5.8 \\
\hline Nominal value & 13,590 & 1379 & & \\
\hline \multicolumn{5}{|l|}{ Method ellipse 2} \\
\hline Absolute mean & 265 & 401 & 27 & 2.2 \\
\hline $\operatorname{Max}$ & 777 & 1060 & 70 & 5.9 \\
\hline Nominal value & 17,903 & 1513 & & \\
\hline
\end{tabular}


Table 4. Restoration angle measurement accuracy.

\begin{tabular}{lccc}
\hline Restoration angle in degree & $3 \mathrm{D}$ & Sagittal & Frontal \\
\hline Mean abs error & 0.9 & 0.6 & 1.2 \\
Standard deviation error & 1.1 & 0.8 & 1.7 \\
Maximal error & 2.3 & 2.2 & 4.1 \\
\hline
\end{tabular}

estimation was $31 \mathrm{~mm}^{3}$ while the $95 \%$ confidence interval was $84 \mathrm{~mm}^{3}$.

\subsubsection{D and 2D height measurement results}

We consider the manual reconstructions as the GS. The mean height differences between GS and 3D-proposed reconstruction of the $\mathrm{L} 1$ vertebra is $0.3 \mathrm{~mm}(\max 0.7 \mathrm{~mm})$ while it is $2.2 \mathrm{~mm}$ ( $\max 5.5 \mathrm{~mm}$ ) for $2 \mathrm{D}$ measurement (Table 6). 2D differences exceed $1 \mathrm{~mm}$ in seven heights out of nine heights.

\section{Discussion}

This study proposed a quantitative 3D approach to estimate the restoration of a fractured vertebra. In order to describe the overall parameters affected by a potential change in global morphometry after a minimally invasive surgery, the above-mentioned measurements involved heights, angles, volumes and region of interest. Even if CT-scan is not radiation free, it is used in clinical routine to check the post- surgery results of kyphoplasty (Spivak and Johnson 2005). Our main concern was focused on developing a precise and robust method, easily reproducible in clinical routine. This methodology was based on semi-automatic 3D reconstructions of the vertebrae from specific CT-scans data (slice thickness $<1 \mathrm{~mm}$ ), with a high image resolution (pixel size $<1 \mathrm{~mm} \times 1 \mathrm{~mm}$ ) and a bone filter applied to the slices. With these fulfilled specifications, which are strict but easily available in clinical routine, 16 3D semi-automatic reconstructions were compared to their respective reference reconstructions, considered as GS; comparisons provided a point-to-surface accuracy inferior to $1 \mathrm{~mm}$, i.e. in the same order of magnitude as the CT-scan slices thickness. Moreover, operator dependency issue, prevailing in the treatment of 2D X-rays, was addressed by running a reproducibility study. Indeed, a manual contribution of the operator appears twice during the process: (1) at the very beginning, where the identification of anatomical landmarks is required and (2) after the initial solution is generated, which needs small adjustments to avoid aberrant $3 \mathrm{D}$ solution. The reproducibility was measured $(1 \mathrm{~mm})$ and considered to be accurate enough to ensure a reliable $3 \mathrm{D}$ object. Therefore, the geometry generated by 3D semiautomatic reconstruction, obtained in a limited amount of manual time $(5 \mathrm{~min})$, could be considered as both reproducible and accurate. As a result, the transformations resulting from a minimally invasive surgery intended for restoring a fractured vertebral body could be described.

Table 5. Local evaluation accuracy results.

\begin{tabular}{lcrrr}
\hline & IC 95\% & Mean & Max & Nominal value \\
\hline Average height restoration per zone $(\mathrm{mm})$ & 1.6 & 0.4 & 2.6 & 2.8 \\
Maximum height restoration per zone (mm) & 1.7 & 0.8 & 3.0 & 2.4 \\
Volume restoration per zone $\left(\mathrm{mm}^{3}\right)$ & 103.0 & 22.0 & 132.0 & 116.0 \\
\hline
\end{tabular}

Table 6. Height measurements of a L1 vertebra using the proposed method and using the method of Genant et al. (1993).

\begin{tabular}{|c|c|c|c|c|c|c|c|c|c|}
\hline & $\begin{array}{l}\text { Anterior } \\
\text { right }\end{array}$ & $\begin{array}{l}\text { Anterior } \\
\text { middle }\end{array}$ & $\begin{array}{l}\text { Anterior } \\
\text { left }\end{array}$ & $\begin{array}{c}\text { Middle } \\
\text { right }\end{array}$ & $\begin{array}{l}\text { Middle } \\
\text { middle }\end{array}$ & $\begin{array}{l}\text { Middle } \\
\text { left }\end{array}$ & $\begin{array}{l}\text { Posterior } \\
\text { right }\end{array}$ & $\begin{array}{l}\text { Posterior } \\
\text { middle }\end{array}$ & $\begin{array}{c}\text { Posterior } \\
\text { left }\end{array}$ \\
\hline \multicolumn{10}{|l|}{ Heights in $\mathrm{mm}$} \\
\hline $\begin{array}{l}\text { Surface measurements on manual recon- } \\
\text { struction }\end{array}$ & 25.2 & 22.7 & 21.3 & 22.9 & 19.0 & 18.5 & 29.4 & 26.3 & 26.7 \\
\hline $\begin{array}{l}\text { Best-fit plane measurements on manual } \\
\text { reconstruction }\end{array}$ & 23.5 & 21.3 & 20.0 & 25.6 & 23.3 & 21.9 & 27.9 & 25.8 & 24.4 \\
\hline $\begin{array}{l}\text { Surface Measurements on 3D Recon- } \\
\text { struction (with proposed method) }\end{array}$ & 25.0 & 22.6 & 21.4 & 23.3 & 19.5 & 18.1 & 29.4 & 27.0 & 26.4 \\
\hline $\begin{array}{l}\text { Best-fit plane measurements on 3D } \\
\text { Reconstruction (with proposed method) }\end{array}$ & 23.5 & 21.1 & 19.8 & 25.8 & 23.4 & 21.8 & 28.2 & 26.1 & 24.4 \\
\hline 2D measurements (Genant et al. 1993) & 19.7 & & & 18.3 & & & 28.0 & & \\
\hline \multicolumn{10}{|l|}{ Errors in $\mathrm{mm}$} \\
\hline $\begin{array}{l}\text { 3D Reconstruction surface measurement } \\
\text { (with proposed method) error }\end{array}$ & -0.2 & -0.1 & 0.1 & 0.4 & 0.5 & -0.4 & 0.0 & 0.7 & -0.3 \\
\hline $\begin{array}{l}\text { 3D Reconstruction best-fit plane } \\
\text { measurement (with proposed method) } \\
\text { error }\end{array}$ & 0.0 & 0.1 & 0.2 & -0.2 & -0.1 & 0.1 & -0.3 & -0.2 & -0.1 \\
\hline 2D measurements error & -5.5 & -3.0 & -1.6 & -4.6 & -0.7 & -0.2 & -1.4 & 1.7 & 1.3 \\
\hline
\end{tabular}


Moreover, in the post-surgical state, the presence of cement and of the implant did not disturb the reconstruction process, as coherence criteria were introduced to overcome the treatment of these foreign body materials composed of densities different from cortical and cancellous bone. In comparison with the existing 3D segmentation process, the quality of the reconstruction was evaluated not only with visual controls (Kaminsky et al. 2004), or by projecting the model in the CT-scan images volume (Mastmeyer et al. 2006) but also with accuracy and reproducibility studies on the point-to-surface distance with the GS 3D vertebrae. For the first time, a semi-automatic process dedicated to the $3 \mathrm{D}$ reconstruction of practical cases such as fractured or restored vertebrae was proposed and evaluated on both lumbar and thoracic spine.

A special care was taken for ensuring the robustness of the proposed parameters, inducing the testing of several possible options. One limitation of this study is that the comparison is made between manual and semi-auto reconstructions, while CT-scan data can yield uncertainty due to partial volume effect. However, GS data for more objective evaluation are hardly available since even direct measurement of isolated vertebrae would provide a bias because the corresponding CT-scan does not provide the same signal as in vivo data. Regarding the grid, semiquantitative technique was derived from the study of Kiel (1995) and Genant et al. (1993), used in 2D lateral view with three points (anterior, middle and posterior) on the superior and inferior endplates, however in this study, the 3D reconstruction allowed to produce height estimation in nine points instead of three. It appeared that for height measurements, the use of a best-fit plane was more reliable than computation done directly on the surface. This technique implies to process nodes that are not exactly located on the surface, but limits the potential errors due to local holes and disturbance generated by the fractures relief of the endplates. Indeed, the fractured vertebral endplates can show a highly erratic shape, with unpredictable local variations that make it difficult to assess reliable and accurate quantification. As a compromise, choosing least square planes for the nine point's projection helps overcoming the issue induced by local pits that may not be representative of the global morphometric aspect of the vertebra. It may be considered as a global indicator of the height restoration, leaving out the local shape abnormalities. This choice yielded a $95 \%$ confidence interval inferior to $1 \mathrm{~mm}$ for heights, while the estimation of restoration difference was evaluated at $1.2 \mathrm{~mm}$. These values may be clinically satisfactory, as in the range of the CT-scan slice thickness. Nevertheless, this accuracy level implies that the quantification evaluation will be more adapted for vertebral restoration higher than $1 \mathrm{~mm}$ : if the recovered height is inferior to that value, the calculation will be within the range of uncertainty.

Furthermore, to illustrate the fact that 3D and 2D height measurements are significantly different, these heights were compared on a L1 vertebra from our vertebrae data-set according to the method of Genant et al. (1993) as illustrated in Figure 9.

If we consider thorough manual reconstructions as the GS, and if we do not consider projection of the endplate surface on a best-fit plane, the mean height differences between GS and 3D proposed reconstruction of the L1 vertebra is $0.3 \mathrm{~mm}(\max 0.7 \mathrm{~mm})$ while it is $2.2 \mathrm{~mm}(\max$ $5.5 \mathrm{~mm}$ ) for 2D measurement (Table 6). Furthermore, 2D differences exceed $1 \mathrm{~mm}$ in seven heights out of nine heights. Genant et al. (1993), which is a reference for 2D measurements, underline that:

However, varying radiographic quality and parallax distortion of the borders of the vertebral body cause many problems in the placement of the points used for digitization. Furthermore, the placement of the points can be done in several ways. The placement is still a subjective "reading" of the x-ray film.

Frontal orientation of the vertebral endplate increases the bias related to $2 \mathrm{D}$ measurement.

Regarding the volume estimation, two types of ellipses were considered to be used as extruded cylinders. The cylinder based on the ellipse 1 was designed to avoid the random irregularities of the vertebral body borders, which makes it less representative than the overall vertebral body volume computed with ellipse 2 . Moreover, ellipse 2 covers an area that is larger than ellipse 1. According to our data-set, the average volume restoration was equivalent

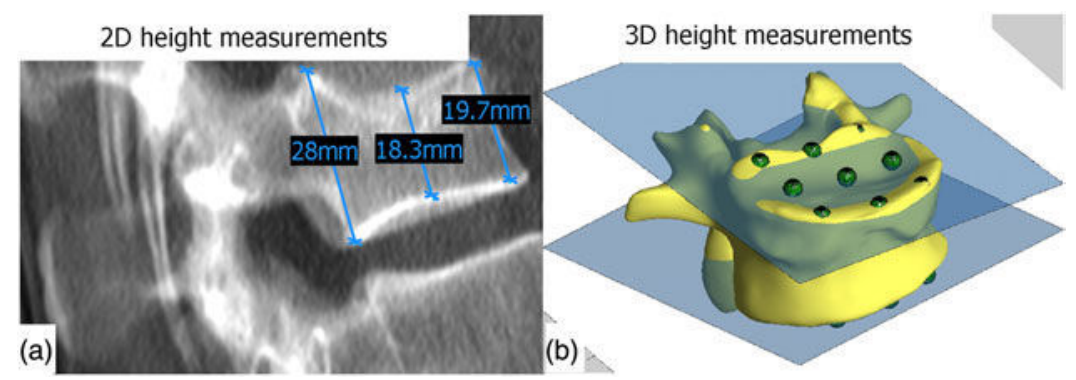

Figure 9. Illustration of height measurements of a L1 vertebra using the proposed method and using the method of Genant et al. (1993). 
using both ellipses $\left(1379 \mathrm{~mm}^{3}\right.$ vs $1513 \mathrm{~mm}^{3}$, respectively, for the volume restoration). This means that, as expected, restoration was mainly located towards the centre of the vertebral body, and not in its periphery. In terms of accuracy, best results were obtained with ellipse 1 . However, ellipse 2 could be more relevant from a clinical point of view and therefore could be an alternative. The error in volume restoration is $324 \mathrm{~mm}^{3}$ which represents $2.4 \%$ of the average nominal global volume value, within the orders of values of the other parameters. The amount of restoration may represent only a small percentage of the global volume, and therefore, the relative uncertainty (ratio error vs restoration volume) gets higher and reaches $23 \%$. This value indicates that the restoration is significant and not included within the uncertainty of measurement. The angles describing the modification of the global orientation of the endplates also provided precise results (about $1^{\circ}$ ). Compared to $2 \mathrm{D}$ estimation where the orientation of the patient can produce large variability depending on the positioning of the $\mathrm{X}$-ray source, the $3 \mathrm{D}$ calculation is more accurate and the angle restoration could be considered as a relevant new clinical index.

Finally, the parameters regarding nine regions of interest were introduced to describe the restoration in a more local aspect, in order to be able to locate the restoration efficiency region by region. This may be useful to discriminate fracture types response to surgery, such as wedge, biconcave and crush.

\section{Conclusion}

In conclusion, in reference to the current state-of-the-art vertebral fracture assessment methods, this study proposed a set of four new reliable measurement methods to evaluate vertebral fractures and surgical restoration in terms of heights, volumes and angles and region of interest. The surgical restoration is now measurable in clinical routine when its impact is at least $1 \mathrm{~mm}$. These measurement methods were based on a new vertebral $3 \mathrm{D}$ reconstruction process. It produces vertebral $3 \mathrm{D}$ solutions with the same magnitude of accuracy $(1 \mathrm{~mm})$ as the CTscan slices thickness. Such method usable in clinical routine should help the assessment of vertebral restoration.

\footnotetext{
Notes

1. Email: lepennecg@gmail.com.

2. Email: sophie.campana@ensam.eu.

3. Email: erwan.jolivet@yahoo.fr.

4. Email: vital.jean-marc@wanadoo.fr.
}

5. Email: xavier.barreau@chu-bordeaux.fr.

6. Email: wafa.skalli@ensam.eu.

\section{References}

Felsenberg D, Silman A, Lunt M, Armbrecht G, Ismail A, Finn J, Cockerill W, Banzer D, Benevolenskaya L, Bhalla A et al., 2002. Incidence of vertebral fracture in europe: results from the European Prospective Osteoporosis Study (EPOS). J Bone Miner Res. 17(4):716-724.

Fitzgibbon A, Pilu M, Robert BF. 1999. Direct least square fitting of ellipses. IEEE Trans Pattern Anal Mach Intell. 21(5):476-480.

Genant HK, Wu CY, Vankuijk C, Nevitt MC. 1993. Vertebral fracture assessment using a semiquantitative technique. J Bone Miner Res. 8(9):1137-1148.

Gold DT. 1996. The clinical impact of vertebral fractures: quality of life in women with osteoporosis. Bone. 18(3):185S-189S.

Grados F, Fechtenbaum J, Flipon E, Kolta S, Roux C, Fardellone P. 2009. Radiographic methods for evaluating osteoporotic vertebral fractures. Joint Bone Spine. 76(3):241-247.

Humbert L, De Guise JA, Aubert B, Godbout B, Skalli W. 2009. $3 \mathrm{D}$ reconstruction of the spine from biplanar X-rays using parametric models based on transversal and longitudinal inferences. Med Eng Phys. 31(6):681-687.

Iso. 1994. Accuracy (Trueness and precision) of measurement methods and results -5725 .

Kaminsky J, Klinge P, Rodt T, Bokemeyer M, Luedemann W, Samii M. 2004. Specially adapted interactive tools for an improved 3D-segmentation of the spine. Comput Med Imaging Graph. 28(3):119-127.

Kiel D. 1995. Assessing vertebral fractures. National Osteoporosis Foundation Working Group on Vertebral Fractures. J Bone Miner Res. 10(4):518-523.

Klinder T, Ostermann J, Ehm M, Franz A, Kneser R, Lorenz C. 2009. Automated model-based vertebra detection, identification, and segmentation in CT images. Med Image Anal. 13(3):471-482.

Laporte S, I DM, Ismael B, Fouchecour MD, Lasseau JP, Lavaste F, Skalli W. 2000. Quantitative morphometric study of thoracic spine. Eur J Orthop Surg Traumatol. 10:85-91.

Laurent CP, Jolivet E, Hodel J, Decq P, Skalli W. 2011. New method for $3 \mathrm{D}$ reconstruction of the human cranial vault from CT-scan data. Med Eng Phys. 33(10):1270-1275.

Mastmeyer A, Engelke K, Fuchs C, Kalender WA. 2006. A hierarchical 3D segmentation method and the definition of vertebral body coordinate systems for QCT of the lumbar spine. Med Image Anal. 10(4):560-577.

Riggs BL, Melton LJ. 1995. The worldwide problem of osteoporosis: insights afforded by epidemiology. Bone. 17(5):505S-511S.

Roth SD. 1982. Ray casting for modeling solids. Comput Graph Image Process. 18(2):109-144.

Semaan I, Skalli W, Veron S, Templier A, Lassau JP, Lavaste F. 2001. Quantitative 3D anatomy of the lumbar spine. Rev Chir Orthop Reparatrice Appar Mot. 87(4):340-353.

Spivak JM, Johnson MG. 2005. Percutaneous treatment of vertebral body pathology. J Am Acad Orthop Surg. 13(1):6-17. 